\title{
Nouvelles recommandations du Guide canadien d'immunisation concernant les pratiques d'administration des vaccins
}

\author{
C Jensen ${ }^{1}$, D Moore ${ }^{2}$, C Mah' ${ }^{3}$, O Baclic ${ }^{1}$, S Marchant-Short ${ }^{4}$ au nom du Comité consultatif national \\ de l'immunisation (CCNI)*
}

\section{Résumé}

Contexte : Le Guide canadien d'immunisation (GCl) est publié en ligne par l'Agence de la santé publique du Canada et résume les directives sur les vaccins à usage humain en une seule ressource. Les chapitres sont révisés et mis à jour régulièrement. L'administration du vaccin est un aspect essentiel de tout programme d'immunisation. Récemment, le chapitre du Guide canadien d'immunisation sur les méthodes d'administration des vaccins a été mis à jour.

Objectif : Fournir les points saillants des récentes modifications apportées au chapitre Méthodes d'administration des vaccins du Guide canadien d'immunisation.

Approche : Les directives propres aux vaccins dans le Guide canadien d'immunisation sont fondées sur les recommandations du Comité consultatif national de l'immunisation (CCNI) et du Comité consultatif de la médecine tropicale et de la médecine des voyages (CCMTMV) ainsi que sur les nouvelles recommandations élaborées par les membres du groupe de travail du Guide canadien d'immunisation et le personnel technique du secrétariat du CCNI. Les nouvelles recommandations sont fondées sur un examen des documents, y compris des examens systématiques, le cas échéant, un examen des conseils fournis par d'autres groupes consultatifs techniques nationaux sur l'immunisation et des avis d'experts. Les révisions sont approuvées par le président du groupe de travail, de même que le CCNI.

Résultats : Les points saillants des nouvelles recommandations sont les suivants : les fournisseurs de vaccins devraient se conformer aux politiques et procédures des administrations ou de l'organisation concernant la combinaison du contenu des flacons multidoses; il faut exercer un jugement clinique lors du choix de la longueur de l'aiguille pour les injections intramusculaires qui tient compte du poids, du sexe et de l'âge du sujet à vacciner; les aiguilles à filtre ne sont pas recommandées pour l'administration du vaccin, car elles peuvent filtrer les ingrédients actifs comme les adjuvants; un autre site d'injection qu'une zone où le drainage lymphatique peut être altéré devrait être envisagé; il n'y a aucune preuve ou justification théorique pour éviter l'injection dans un tatouage ou une tache de naissance superficielle; des stratégies de gestion de la douleur d'immunisation ont maintenant été élaborées pour tous les âges.

Conclusion : Les recommandations concernant les méthodes d'administration des vaccins ont récemment été modifiées de façon importante. L'Agence de la santé publique du Canada s'est engagée à fournir des renseignements concernant l'immunisation dans un format facilement accessible et lisible pour les fournisseurs de soins de santé et les responsables des politiques.

\section{Affiliations}

${ }^{1}$ Centre de l'immunisation et des maladies respiratoires infectieuses, Agence de la santé publique du Canada, Ottawa (Ontario)

${ }^{2}$ Université McGill, Montréal (Québec)

${ }^{3}$ Université Dalhousie, Halifax (Nouvelle-Écosse) et Université de Toronto, Toronto (Ontario)

${ }^{4}$ Soins infirmiers en santé publique, Santé Î.-P.-É., Charlottetown (Î.-P.-É.)

*Correspondance : naci-ccni@ phac-aspc.gc.ca

Citation proposée : Jensen C, Moore D, Mah C, Baclic O, Marchant-Short S, au nom du Comité consultatif national de l'immunisation (CCNI). Nouvelles recommandations du Guide canadien d'immunisation concernant les pratiques d'administration des vaccins. Relevé des maladies transmissibles au Canada. 2017;43(11):276-8. https://doi.org/10.14745/ccdr.v43i11a06f

\section{Introduction}

Le Guide canadien d'immunisation ( $\mathrm{GCl}$ ) est publié en ligne par I'Agence de la santé publique du Canada (ASPC). Depuis 1979, il s'agit d'un résumé fiable et facile à lire de renseignements sur l'immunisation pour les fournisseurs de soins de santé qui administrent des vaccins à leurs patients et pour les responsables des politiques pour la prestation de programmes d'immunisation (1). Le Guide canadien d'immunisation est divisé en cinq parties et résume les recommandations et les conseils du Comité consultatif national de l'immunisation (CCNI) et du Comité consultatif de la médecine tropicale et de la médecine des voyages (CCMTMV). Les chapitres sont révisés et mis à jour tous les quatre ans ou plus souvent en cas de nouvelle recommandation ou de changement de pratique. Un tableau des 
mises à jour (2) résume les principales modifications apportées aux différents chapitres.

L'administration du vaccin est un aspect essentiel de tout programme d'immunisation. Les méthodes d'administration des vaccins tiennent compte d'importants facteurs comme la consultation avant la vaccination, la préparation du vaccin et le choix de l'aiguille, ainsi que la détermination de la bonne voie, du bon site et de la bonne technique d'administration du vaccin. Les stratégies de gestion de la douleur, de la consultation et de l'observation après la vaccination, ainsi que la prévention et le contrôle des infections font également partie intégrante des méthodes d'administration des vaccins. L'objectif de cet article est de présenter les points saillants des principales modifications apportées au chapitre "Méthodes d'administration des vaccins » de la partie 1 du Guide canadien d'immunisation.

\section{Approche}

Les directives propres aux vaccins qui figurent dans le Guide canadien d'immunisation sont résumées à partir des recommandations du CCNI et du CCMTMV telles que rédigées dans les énoncés et les mises à jour (3),(4). Les nouvelles recommandations qui ne figurent pas dans un énoncé du CCNI ou du CCMTMV sont rédigées par les membres du groupe de travail du Guide canadien d'immunisation et le personnel technique du secrétariat du CCNI de I'ASPC. Les révisions sont approuvées par le président du groupe de travail, de même que le CCNI. Le groupe de travail de la partie 1 a été mis sur pied pour réviser le chapitre Méthodes d'administration des vaccins en août 2016. La version précédente du chapitre Méthodes $\mathrm{d}^{\prime}$ administration des vaccins a été publiée en ligne en 2014. Une révision du chapitre a été entreprise avant le cycle de quatre ans, car de nouvelles recherches ont été menées, en particulier dans les domaines de la sélection des aiguilles et de la gestion de la douleur, ce qui a donné lieu à une révision du chapitre.

Une révision complète du chapitre a été entreprise. Le membre de liaison du CCNI du Comité canadien d'immunisation a sollicité la rétroaction des immunisateurs infirmiers sur le chapitre existant. Les demandes de précisions et d'orientation ont été examinées et le chapitre a été examiné afin de cerner les questions qui nécessitaient un examen des documents. Les recommandations révisées sur la longueur de l'aiguille présentées dans le «tableau 3 : Lignes directrices pour le choix d'une aiguille » du chapitre Méthodes d'administration des vaccins étaient fondées sur un examen des documents, un examen des conseils fournis par d'autres groupes consultatifs techniques nationaux sur l'immunisation (GCNTV) et des opinions d'experts. Les recommandations révisées du « tableau 4 : Stratégies de gestion de la douleur associée à la vaccination pour les enfants, en fonction des groupes d'âge » du chapitre "Méthodes d'administration des vaccins » étaient fondées sur l'examen systématique des stratégies de réduction de la douleur liée à l'injection de vaccins de Taddio et ses collègues (5). Les six examens systématiques, sur lesquels les lignes directrices étaient fondées, ont été évalués à l'aide de la liste de contrôle AMSTAR (évaluation méthodologique de la qualité des examens systématiques) établie par le secrétariat du CCNI avant d'être inclus dans la révision du chapitre (6).

\section{Résumé des mises à jour}

Les mises à jour sont résumées dans le tableau 1. Pour obtenir des renseignements complets, veuillez consulter le chapitre Méthodes d'administration des vaccins du Guide canadien d'immunisation (7).

\section{Tableau 1 : Principales modifications apportées au chapitre " Méthodes d'administration des vaccins "}

\begin{tabular}{|c|c|c|c|}
\hline Sujet & $\begin{array}{c}\text { En-tête de } \\
\text { section - } \\
\text { Sous-rubrique }\end{array}$ & $\begin{array}{l}\text { Lignes } \\
\text { directrices } \\
\text { précédentes }\end{array}$ & $\begin{array}{l}\text { Lignes directrices } \\
\text { révisées ou } \\
\text { nouvelles }\end{array}$ \\
\hline $\begin{array}{l}\text { Combinaison } \\
\text { du contenu } \\
\text { des flacons } \\
\text { multidoses }\end{array}$ & $\begin{array}{l}\text { Administration } \\
\text { des vaccins } \\
\text { Préparation } \\
\text { vaccinale } \\
\text { Inspection } \\
\text { du vaccin et } \\
\text { mélange }\end{array}$ & $\begin{array}{l}\text { Lors des } \\
\text { cliniques de } \\
\text { vaccination } \\
\text { dans le cadre } \\
\text { desquelles un } \\
\text { seul vaccin est } \\
\text { administré, } \\
\text { le contenu } \\
\text { de plusieurs } \\
\text { flacons } \\
\text { multidoses } \\
\text { peut être } \\
\text { combiné, } \\
\text { pour éviter } \\
\text { le gaspillage, } \\
\text { si les flacons } \\
\text { ont le même } \\
\text { numéro de lot. }\end{array}$ & $\begin{array}{l}\text { Les vaccinateurs doivent } \\
\text { se conformer aux } \\
\text { politiques et procédures } \\
\text { juridictionnelles ou } \\
\text { organisationnelles } \\
\text { concernant la } \\
\text { combinaison du contenu } \\
\text { de flacons multidoses. }\end{array}$ \\
\hline $\begin{array}{l}\text { Choix de } \\
\text { l'aiguille pour } \\
\text { les injections } \\
\text { intramusculaires } \\
\text { (IM) }\end{array}$ & $\begin{array}{l}\text { Administration } \\
\text { des vaccins } \\
\text { Choix de } \\
\text { l'aiguille } \\
\text { Tableau } 3 \text { : } \\
\text { Lignes directrices } \\
\text { pour le choix } \\
\text { d'une aiguille } \\
\text { Voie } \\
\text { d'administration, } \\
\text { voie } \\
\text { intramusculaire } \\
\text { (IM) }\end{array}$ & $\begin{array}{l}\text { Longueur de } \\
\text { l'aiguille : } \\
2,2 \text { à } 2,5 \mathrm{~cm} \\
\text { (7/8 à } 1 \text { po) pour } \\
\text { les nourrissons, } \\
\text { les tout-petits } \\
\text { et les enfants } \\
\text { plus âgés; } \\
2,5 \text { à } 3,8 \mathrm{~cm} \\
\text { (1 à } 111 / 2 \text { po) } \\
\text { pour les } \\
\text { adolescents et } \\
\text { les adultes. }\end{array}$ & $\begin{array}{l}\text { Le tableau } 3 \text { révisé } \\
\text { fournit une gamme } \\
\text { de longueurs } \\
\text { d'aiguille puisque } \\
\text { le choix de l'aiguille } \\
\text { pour les injections } \\
\text { intramusculaires doit } \\
\text { être fondé sur le } \\
\text { jugement clinique. II } \\
\text { faut tenir compte du } \\
\text { poids, du sexe et de } \\
\text { l'âge du sujet à vacciner. }\end{array}$ \\
\hline $\begin{array}{l}\text { Utilisation } \\
\text { d'aiguilles à } \\
\text { filtre }\end{array}$ & $\begin{array}{l}\text { Administration } \\
\text { des vaccins } \\
\text { Choix de } \\
\text { l'aiguille }\end{array}$ & Aucune & $\begin{array}{l}\text { Les aiguilles à filtre ne } \\
\text { sont pas recommandées } \\
\text { pour l'administration } \\
\text { des vaccins, car elles } \\
\text { peuvent filtrer les } \\
\text { ingrédients actifs } \\
\text { comme des adjuvants. }\end{array}$ \\
\hline $\begin{array}{l}\text { Administration } \\
\text { du vaccin dans } \\
\text { une zone où } \\
\text { la circulation } \\
\text { lymphatique } \\
\text { peut être } \\
\text { altérée ou dans } \\
\text { un tatouage } \\
\text { ou une tache } \\
\text { de naissance } \\
\text { superficielle }\end{array}$ & $\begin{array}{l}\text { Administration } \\
\text { des vaccins } \\
\text { Voies et } \\
\text { méthodes } \\
\text { d'administration } \\
\text { des vaccins, sites } \\
\text { d'injection } \\
\text { Vaccins à } \\
\text { administration } \\
\text { parentérale }\end{array}$ & Aucune & $\begin{array}{l}\text { L'injection d'un vaccin } \\
\text { dans une zone où la } \\
\text { circulation lymphatique } \\
\text { peut être une réduite } \\
\text { (p. ex., lymphœdème } \\
\text { local, lymphangiome, } \\
\text { dissection du ganglion } \\
\text { axillaire, fistule } \\
\text { artério-veineuse (A-V), } \\
\text { amputation d'un } \\
\text { membre supérieur) } \\
\text { pourrait théoriquement } \\
\text { entraîner une réponse } \\
\text { immunitaire affaiblie en } \\
\text { raison d'une absorption } \\
\text { réduite du vaccin, bien } \\
\text { qu'il n'existe pas de } \\
\text { données à l'appui. } \\
\text { Envisagez un autre } \\
\text { site d'injection, dans la } \\
\text { mesure du possible. Il } \\
\text { n'y a pas de données } \\
\text { probantes ou de }\end{array}$ \\
\hline
\end{tabular}


Tableau 1 : Principales modifications apportées au chapitre "Méthodes d'administration des vaccins" (suite)

\begin{tabular}{|c|c|c|c|}
\hline Sujet & $\begin{array}{l}\text { En-tête de } \\
\text { section } \\
\text { - Sous- } \\
\text { rubrique }\end{array}$ & $\begin{array}{l}\text { Lignes } \\
\text { directrices } \\
\text { précédentes }\end{array}$ & $\begin{array}{l}\text { Lignes directrices } \\
\text { révisées ou } \\
\text { nouvelles }\end{array}$ \\
\hline & & & $\begin{array}{l}\text { justification théorique } \\
\text { pour éviter l'injection } \\
\text { dans un tatouage ou } \\
\text { une tache de naissance } \\
\text { superficielle. }\end{array}$ \\
\hline $\begin{array}{l}\text { Techniques } \\
\text { pour atténuer } \\
\text { la douleur } \\
\text { causée par les } \\
\text { injections de } \\
\text { vaccin }\end{array}$ & $\begin{array}{l}\text { Administration } \\
\text { des vaccins } \\
\text { Techniques } \\
\text { pour atténuer la } \\
\text { douleur causée } \\
\text { par les injections } \\
\text { de vaccin } \\
\text { Tableau } 4 \text { : } \\
\text { Stratégies de } \\
\text { gestion de la } \\
\text { douleur associée } \\
\text { à la vaccination } \\
\text { pour les enfants, } \\
\text { en fonction des } \\
\text { groupes d'âge }\end{array}$ & $\begin{array}{l}\text { La version } \\
\text { précédente } \\
\text { du tableau } 4 \\
\text { ne comportait } \\
\text { que des } \\
\text { stratégies de } \\
\text { gestion de la } \\
\text { douleur liée à } \\
\text { l'immunisation } \\
\text { chez les } \\
\text { enfants. }\end{array}$ & $\begin{array}{l}\text { La version révisée du } \\
\text { tableau } 4 \text { fournit des } \\
\text { stratégies de gestion } \\
\text { de la douleur liée à } \\
\text { l'immunisation pour } \\
\text { tous les âges. }\end{array}$ \\
\hline
\end{tabular}

\section{Discussion}

Les recommandations concernant les méthodes d'administration des vaccins ont changé récemment de façon importante; les fournisseurs de vaccins au Canada devraient consulter les politiques des administrations ou des organisations au sujet de la combinaison du contenu des flacons multidoses. On encourage désormais les fournisseurs de vaccins à tenir compte du poids, du sexe et de l'âge dans le choix de la longueur des aiguilles pour les injections intramusculaires. En outre, il existe maintenant des stratégies de gestion de la douleur pour diminuer la douleur liée à l'injection pour tous les âges. Ces nouvelles recommandations pourraient éclairer les prochaines campagnes de vaccination sur le plan des méthodes d'administration des vaccins.

L'Agence de la santé publique du Canada s'engage à fournir des renseignements concernant l'immunisation et les vaccins offerts au Canada dans un format facilement accessible et lisible grâce à des mises à jour opportunes et suivies du Guide canadien d'immunisation. Pour obtenir des renseignements concernant les nouvelles recommandations, déclarations et mises à jour du CCNI, ainsi que sur les mises à jour des chapitres du Guide canadien d'immunisation, veuillez vous abonner à la liste de diffusion (8).

\section{Déclaration des auteurs}

Ce résumé de rapports a été rédigé par le groupe de travail de la partie 1 du Guide canadien d'immunisation: S. Marchant-Short (président), D. Moore, C. Mah, C. Jensen et O. Baclic.

S. Marchant-Short est un membre du CCNI. D. Moore et C. Mah sont membres de liaison du CCNI.

\section{Conflit d'intérêt}

Aucun.

\section{Remerciements}

Les auteurs tiennent à remercier les membres extrêmement dévoués du CCNI, ainsi que le personnel de l'Agence de la santé publique du Canada pour leur soutien dans le cadre du processus du Guide canadien d'immunisation.

\section{Financement}

L'Agence de la santé publique du Canada soutient les activités du CCNI en tant qu'organisme consultatif externe.

\section{Références}

1. Gouvernement du Canada. Guide canadien d'immunisation. Ottawa ON: ASCP. https://www.canada.ca/fr/sante-publique/ services/guide-canadien-immunisation.html [Consulté le 8 septembre 2017].

2. Gouvernement du Canada. Guide canadien d'immunisation : Mises à jour des chapitres. Ottawa ON. https://www.canada.ca/ $\mathrm{fr} /$ sante-publique/services/guide-canadien-immunisation/misesa-jour-chapitres.html [Consulté le 8 septembre 2017].

3. Gouvernement du Canada. Comité consultatif national de I'immunisation. Ottawa ON: ASPC. https://www.canada.ca/ $\mathrm{fr} /$ sante-publique/services/immunisation/comite-consultatifnational-immunisation-ccni.html [Consulté le 8 septembre 2017].

4. Gouvernement du Canada. Qu'est-ce que le CCMTMV? Ottawa ON: ASPC. https://www.canada.ca/fr/sante-publique/services/santevoyageurs/est-ccmtmv.html [Consulté le 8 septembre 2017].

5. Taddio A, McMurtry CM, Shah V, Riddell RP, Chambers CT, Noel M, MacDonald NE, Rogers J, Bucci LM, Mousmanis $P$, Lang E, Halperin SA, Bowles S, Halpert C, Ipp M, Asmundson GJG, Rieder MJ, Robson K, Uleryk E, Antony MM, Dubey V, Hanrahan A, Lockett D, Scott J, Bleeker EV; HELPinKIDS\&Adults. Reducing pain during vaccine injections: clinical practice guideline. CMAJ 2015; 187(13):975-82. DOI (http://dx.doi. org/10.1503/cmaj.150391). PubMed (https://www.ncbi.nlm. nih.gov/entrez/query.fcgi?cmd=Retrieve \&db=PubMed\&lis t_uids=26303247\&dopt=Abstract).

6. Shea BJ, Grimshaw JM, Wells GA, Boers M, Andersson N, Hamel C, Porter AC, Tugwell P, Moher D, Bouter LM. Development of AMSTAR: a measurement tool to assess the methodological quality of systematic reviews. BMC Medical Research Methodology. 2007;7(10):1-7. DOI (https://doi. org/10.1186/1471-2288-7-10). PubMed (https://www.ncbi. $\mathrm{nlm}$.nih.gov/entrez/query.fcgi?cmd=Retrieve\&db=PubMed\&li st_uids=17302989\&dopt=Abstract).

7. Gouvernement du Canada. Guide canadien d'immunisation : Partie 1 - Information clé sur l'immunisation. Méthodes d'administration des vaccins. Ottawa ON. https://www.canada. $\mathrm{ca} / \mathrm{fr} /$ sante-publique/services/publications/vie-saine/guidecanadien-immunisation-partie-1-information-cle-immunisation/ page-8-methodes-administration-vaccins.html [Consulté le 8 septembre 2017].

8. Gouvernement du Canada. Mises à jour du Guide canadien d'immunisation et publication du Comité consultatif national d'immunisation - Liste de diffusion. Ottawa ON. http:// www.canadiensensante.gc.ca/healthy-living-vie-saine/ immunization-immunisation/canadian-immunization-guidecanadien-immunisation/email-subscription-abonnementcourriel-fra.php?_ga=2.36853792.187965983.1508431953994711086.1501004754 [Consulté le 8 septembre 2017]. 